\section{Christoph Bachmann}

\section{Unterschiede und \\ Gemeinsamkeiten}

$\mathrm{Zu}$ den komplementärmedizinischen Therapierichtungen, die mit Pflanzen arbeiten, gehören die Phytotherapie, die Spagyrik, die Gemmotherapie, die Bachblütentherapie sowie die Klassische Homöopathie, die nebst Mineralien, Tiergiften usw. auch viele Pflanzen verwenden. Jacqueline Ryffel weist auf die unterschiedlichen Therapieanwendungen dieser Methoden hin, die sich aber vor allem sehr deutlich in der Herstellung unterscheiden.

\section{Phytotherapie}

In der Phytotherapie wird zuerst eine Tinktur hergestellt. Dazu wird die meist getrocknete Arzneipflanze mit Alkohol versetzt und mehrere Tage lang mazeriert. Der Alkohol extrahiert Sekundärmetaboliten der Arzneipflanze, die später eine pharmakologische bzw. therapeutische Wirkung erzielen. Wenn z.B. Papaver somniferum (Schlafmohn) mazeriert wird, dann findet man im Mazerat Alkaloide, deren wichtigstes Papaverin ist. Nach dem Entfernen der ausgezogenen Arzneipflanze kann man die alkoholische Lösung als Opium-Tinktur einsetzen oder den Alkohol abdampfen und den Trockenextrakt als feste Arzneiform verwenden.

\section{Spagyrik}

Die Spagyrik schlägt einen anderen Weg ein. Um die Inhaltsstoffe zu ex-

\title{
Jacqueline Ryffel - Geschäftsführerin und VR-Präsidentin der Spagyros AG: Breite Erfahrung in pflanzlicher Komplementärmedizin
}

Jacqueline Ryffel (Kasten 1), Geschäftsführerin der Spagyros, hat nach ihrem Pharmaziestudium eher zufälligerweise einen Homöopathie-Kurs von Dr. med. Martin Furlenmeier (Kasten 2) besucht. Dies hat ihr ganzes Berufsleben nachhaltig geprägt. Heute besitzt sie eine breite Kompetenz in der Herstellung und Anwendung von komplementärmedizinischen Methoden, die Pflanzen einsetzen. In einem Gespräch mit der SCHWEIZERISCHEN ZeITSCHRIFT FüR GanZHeITSMEDIZIN vergleicht sie die verschiedenen Therapierichtungen.

Kasten 1. Jacqueline Ryffel

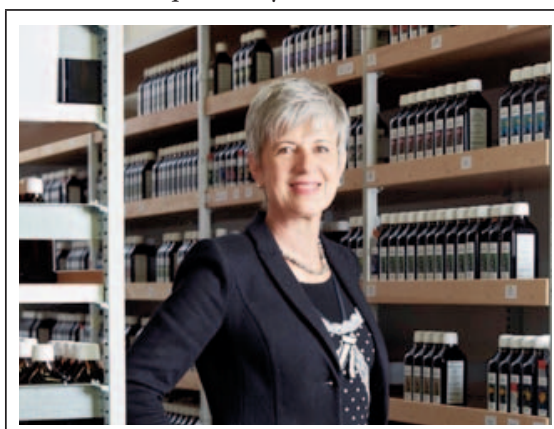

Dipl. pharm. Jacqueline Ryffel studierte Pharmazie an der Universität Bern und begann anschliessend immer mehr, sich komplementärmedizinisch auszubilden und zu betätigen. Heute trägt sie den FPH-Titel Klassische Homöopathie und ist Geschäftsführerin sowie Verwaltungsratspräsidentin der Spagyros AG. Diese Firma stellt Präparate in Klassischer Homöopathie, Spagyrik, Gemmotherapie und Phytotherapie her. Privat ist Frau Ryffel eine begeisterte Hobbyköchin und joggt gern. Sie ist Mitbegründerin des Schweizer Frauenlauf Bern.

trahieren, wird die ganze Arzneipflanze inklusive Wurzel nicht mit Alkohol versetzt, sondern man unterzieht die Arzneipflanze einer alkoholischen Gärung mit Hefepilzen und Zucker. Der dabei entstehende Alkohol löst Inhaltsstoffe besonders tiefgreifend, und
Kasten 2. Dr. med. Martin Furlenmeier (1932-1994)

Der Arzt Martin Furlenmeier hat Jacqueline Ryffel viele Impulse verliehen. Sie betont im Gespräch, dass sie ihm ihr jetziges Wissen über und ihre Einstellung zur Komplementärmedizin verdanke. Furlenmeier hatte vor dem Medizinstudium schon Mathematik, Physik und Botanik studiert. Er war also in verschiedenen Wissenschaften bewandert und wandte trotzdem auch sogenannte unwissenschaftliche Methoden an. In seinem ersten Buch «Kraft der Heilpflanzen» erklärt Furlenmeier seine Vorgehensweise: «Obwohl ich darin nicht die herkömmlichen Wege der Wissenschaft beschreite, möchte ich betonen, dass ich keineswegs ein Gegner der Wissenschaft bin - ganz im Gegenteil!» Er betont, dass verschiedene Therapieformen «ihre volle Berechtigung haben, wenn sich der Praktizierende der Indikationen und Grenzen (...) (der (erg.)) Methoden bewusst ist».

gleichzeitig werden toxische Stoffe abgebaut. Nach dem abgeschlossenen Gärprozess, der 3-10 Wochen dauern kann, wird filtriert. Die Maische wird destilliert und die übrig bleibenden Pflanzenteile werden verbrannt und bei $450{ }^{\circ} \mathrm{C}$ kalziniert (dadurch lassen sich die Mineralsalze der Pflanzen gewinnen). Am Schluss wird wieder alles zusammengefügt und vereint. Diese drei Vorgänge, d.h. Gärung, Destilla-

\section{KARGER}

(c) 2017 S. Karger GmbH, Freiburg

Fax +497614520714 
tion und Verbrennung, führen - etwas vereinfacht ausgedrückt - zur Darstellung der drei verschiedenen Prinzipien. Dabei handelt es sich um philosophische Prinzipien, die hier nicht näher erklärt werden sollen. Giftstoffe, wie giftige Alkaloide, liegen in einer spagyrischen Lösung nicht mehr in toxischen Konzentrationen vor, sondern in einer Konzentration, die etwa einer homöopathischen Potenz von D4 bis D8 entspricht; dafür enthält eine spagyrische Essenz die wichtigsten natürlichen Mineralsalze. Insgesamt geht es in der Spagyrik aber genauso um die feinstofflichen Anteile der Pflanze wie um ihre Wirkstoffe.

Die Herstellung eines spagyrischen Präparates ist ein sehr vielschichtiger und langwieriger Prozess. Vom Moment der Ernte bis zur Fertigstellung einer spagyrischen Essenz dauert es etwa 3 Monate.

\section{Homöopathie}

Noch eine andere Verarbeitungsart für Pflanzen kennt die Homöopathie. Homöopathische Potenzen können aus einer Urtinktur oder einer Verreibung hergestellt werden. Die Firma Spagyros verreibt bis zur 6. Potenz mit Milchzucker, danach wird von Hand in Mehrglasmethode bis zur 200. Potenz mit Alkohol verdünnt und verschüttelt (= potenziert).

\section{Gemmotherapie}

In der Gemmotherapie werden keine Blätter, Früchte oder Wurzeln verarbeitet, sondern die Knospen von Bäumen und Sträuchern. Diese spriessen im Frühjahr und enthalten Wachstumshormone, viele Aminosäuren und andere Proteinbausteine. Bei einer Alkoholmazeration würden diese Inhaltsstoffe denaturiert. Deshalb wird für die Auszüge des Gemmomazerates ein Ethanol-Glyzerin-Gemisch verwendet. Dadurch wird die Polarität der Wasserstoffbrücken verändert. Diese Inhaltsstoffe sind auch nach der Extraktion als Aminosäuren und Pro- teinbaustoffe nachweisbar. Andere Inhaltsstoffe, z.B. zum Teil giftige Sekundärmetabolite der Pflanzenorgane wie Alkaloide, Glykoside usw., werden erst später voll ausgebildet und sind in den Knospen nicht oder in sehr geringer Konzentration enthalten.

Jacqueline Ryffel betont, dass dies der Aspekt ist, der diese Knospenmazerate so wertvoll macht, weil sie relativ viele nachweisbare Stoffe enthalten, und sagt wörtlich: «Man findet nebst den Aminosäuren auch gewisse Flavonoide, Pflanzenwachstumshormone, Enzyme und Vitamin C. Die Gemmotherapie liegt also irgendwie zwischen einer Urtinktur, die stofflich wirkt, und zwischen der Spagyrik, deren Wirkung sich im Feinstofflichen bewegt.»

\section{Der Vergleich mit einem Haus}

Jacqueline Ryffel vergleicht die vier Methoden mit einem Haus:

- Die Phytotherapie bildet das Fundament. Hier geht es um den Wirkstoff, vor allem um sekundäre Pflanzenstoffe. Diese erzeugen im Organismus eine pharmakologische Wirkung.

- Die Gemmotherapie stellt das Erdgeschoss dar. Hier geht es vorwiegend um die primären Pflanzenstoffe wie Aminosäuren, Proteine und Vitamine. Diese wirken auf der funktionalen Ebene und beeinflussen die Albumin- und GlobulinKonzentration im Blut.

- Die Spagyrik ist der erste Stock des Hauses. Hier geht es um das Wirkprinzip, um Informationen, welche durch die Verarbeitung für den Menschen nutzbar gemacht wurden.

- Die Klassische Homöopathie bildet das Dach des Hauses. Hier geht es um die Wirkkraft und um die Feinstofflichkeit, um energetische Schwingungen.

\section{Was entsteht?}

Durch die unterschiedliche Aufbereitungsart der Pflanze oder ihrer Teile entstehen ganz unterschiedliche Arzneien, welche sehr differenziert zum Einsatz kommen.

So sind es in der Phytotherapie vor allem akute Erkrankungen wie Erkältung, Fieber, Husten, akute Blasenentzündung usw., die mit den Pflanzenextrakten Linderung erfahren. Die Therapie basiert zum Teil auf klinischen Studien, aber auch auf der Erfahrungsheilkunde.

Die Gemmomazerate beeinflussen die indirekte und die direkte Immunabwehr. Dadurch werden sie bei Infektionskrankheiten, Allergien und Hauterkrankungen sowie zum Entschlacken und zur Grundgewebereinigung eingesetzt.

Spagyrische Arzneien zeigen ihr vollstes Wirkpotenzial nebst den akuten Erkrankungen vor allem bei langwierigen, chronischen Krankheiten und in der Rekonvaleszenz, bei $\mathrm{Zu}$ ständen, die auf Energiemangel zurückzuführen sind, bei Burnout und depressiven Verstimmungen sowie bei starker körperlicher und nervlicher Beanspruchung. Dank den enthaltenen Mineralien erhält der Körper wieder Halt.

Die homöopathischen Arzneien vermögen die Selbstheilungskräfte des Menschen anzuregen. Sie werden überall dort eingesetzt, wo andere Therapieformen anstossen oder versagen, z.B. bei multiplen Symptomen, die über die Symptome eines spezifischen Krankheitsbildes hinausgehen.

\section{Homöopathische Wirkung}

Jacqueline Ryffels Modell der homöopathischen Wirkung sieht folgendermassen aus: Der Körper eines Menschen besteht zu einem grossen Teil aus Wasser, zu etwa $80-90 \%$. Wasser besteht aus Wassermolekülen, d.h. je aus einem Sauerstoff- und zwei Wasserstoffatomen. Diese sind ener- 
getisch miteinander verbunden und schwingen. Sie sind nicht starr, sondern bewegen sich. Die homöopathischen Potenzen könnten die Schwingungsfrequenzen dieser Sauerstoff-Wasserstoff-Gebilde leicht beeinflussen. Und eine solche homöopathische Information, also die Beeinflussung derSchwingfrequenz, geht blitzschnell durch den Körper. Ein solcher Impuls löst in unserem Körper einen bisher noch unbekannten Vorgang aus, der eine Heilwirkung anregen kann.

\section{Wirkungsweise in der Spagyrik und in der Gemmotherapie}

Bei diesen beiden Methoden gibt es gemäss Jacqueline Ryffel verschiedene Wirkungsmechanismen, weil beide Methoden noch einen Anteil an stofflichen Wirkprinzipien besitzen - in der Gemmotherapie noch mehr als in der Spagyrik. Und dieses Stoffliche an der Gemmotherapie kann im Körper bestimmt eine gewisse pharmakologische Wirkung auslösen, denn die Pharmakologie spielt sich nicht nur in hohen Dosen ab. Auch kleine Dosen können pharmakologische Impulse auslösen.

«Die heutige Medizin forscht auf der Ebene der Nanopartikel. Das ist doch genau die Ebene, auf der sich Wirkprinzipien von Homöopathie, Spagyrik und Gemmotherapie abspielen! Und die heutige Medizin forscht auf Biologics, Frischzellen und Frischpflanzen. Das ist doch nichts anderes, als auf alten Traditionen aufzubauen und neu zu interpretieren!»

\section{Synergistische Wirkungen}

Weiter betont die Geschäftsführerin der Spagyros AG, dass sich die verschiedenen pflanzlichen Therapierichtungen gegenseitig gut ergänzen. Das Zusammenspiel der heilenden Kräfte von Baum, Strauch und Kraut wäre ideal. Aus Knospen der Bäume und Sträucher stellt man Gemmo-
Arzneien und aus Kräutern Tinkturen und spagyrisches Essen her. Man weiss, dass für gewisse Krankheiten die eine Arzneipflanze gut ist und für andere Krankheitsbilder eine andere. Wenn man dem Körper im Falle einer Krankheit eine bestimmte Arzneipflanze als spagyrische Zubereitung verabreicht und eine andere Pflanze als Gemmomazerat zuführt, dann wirken diese auf verschiedenen Ebenen, die sich nicht stören, sondern ergänzen. Wir können also unseren Körper auf diesen verschiedenen Ebenen therapieren. Wenn wir z.B. starke körperliche Symptome haben, die auch geistige oder psychische Beschwerden auslösen können, dann liegt ein komplexes Krankheitsbild vor. Dieses muss mit stofflichen sowie mit feinstofflichen Wirkprinzipien behandelt werden. Je komplexer ein Krankheitsbild ist, umso mehr spricht dies für eine homöopathische Therapie. Wenn jemand Ängste hat und diese Ängste Magenprobleme, z.B. Hyperazidität, auslösen, dann nützt eine Behandlung mit Protonenpumpeninhibitoren (PPI) nichts, weil die Ängste die Hyperazidität immer wieder neu auslösen.

\section{Individualität in der Behandlung}

Jacqueline Ryffel drückt klar aus, dass bei einer bestehenden Erkrankung die Auswahl der Therapiemethode sehr individuell ist. Und das hängt stark vom Therapeuten ab. Jeder hat eine Vorliebe für die eine oder andere Methode und eine Hauptausbildung. Und dies ist gemäss Ryffel das wichtigste Kriterium für die Auswahl der einen oder anderen Therapierichtung. Er muss von der eingesetzten Methode überzeugt sein.

Ein anderer Therapeut würde in einem vorliegenden Fall vielleicht eine andere Therapiemethode einsetzen, weil er von dieser anderen Methode überzeugt ist. Und beide Therapeuten können beim selben Patienten und denselben Beschwerden mit ihren un- terschiedlichen Methoden Erfolg haben. Der Patient ist ein Individuum, der Therapeut ist ein Individuum und dazwischen muss es eine grosse Empathie geben, damit eine Behandlungsweise zum Erfolg führen kann.

Wenn nun z.B. eine Frau Menstruationsbeschwerden hat, dann behandelt man diese vorzugsweise mit Urtinkturen oder Gemmotherapie, weil Mensbeschwerden und diese beiden Therapierichtungen vor allem im körperlichen Bereich angesiedelt sind.

Man kann hinter diesen Mensbeschwerden aber noch eine weitere Dimension sehen und sich fragen, warum diese Frau während jeder Menstruation Krämpfe hat. Ist sie erschöpft und müde, liegt eine Endometriose vor oder bestehen Kummer und Sorgen? In dem Fall würde man dann bei der Spagyrik suchen oder eine homöopathische Anamnese aufnehmen.

\section{Befragung wichtig}

Genau hier weist Jacqueline Ryffel auf einen wichtigen Punkt hin: Bei einer homöopathischen Behandlung muss der Patient sich selber sehr genau beobachten und dem Homöopath bzw. der Homöopathin (hier stellvertretend für alle, die eine fundierte Ausbildung darin haben, unabhängig $\mathrm{ob}$ Arzt/Ärztin, Apotheker/Apothekerin oder Heilpraktiker/Heilpraktikerin) seine Symptome sehr exakt beschreiben, denn nur so wird es möglich sein, in der Repertorisation das sogenannte Simile, die auf die Symptome des Patienten abgestimmte homöopathische Arznei, herauszufinden. Wenn in einer solchen Behandlung der Patient mit Migräne nur aussagen kann, er habe Kopfweh, er aber nicht in der Lage ist, nähere Umstände über die Art der Kopfschmerzen (Klopfen, Hämmern, Drücken, Brennen usw.) anzugeben und auch nicht über Modalitäten berichtet, die eine Verbesserung oder Verschlechterung bewirken bzw. über die ganzen Begleitsymptome wie Übelkeit, Schwindel, Verlan- 
gen nach Dunkelheit, dann kann der Homöopath keine seriöse Repertorisation vornehmen und somit auch keine homöopathische Arznei zur Behandlung dieser Kopfschmerzen verordnen. In einem solchen Moment ist es möglicherweise sinnvoller, vorerst eine Phytoarznei, z.B. Silybum-marianum-Tinktur zu verordnen, wenn die wenigen Aussagen des Patienten vermuten lassen, dass diesen Kopfschmerzen eventuell eine Leberbelastung zugrunde liegen könnte.

\section{Feinstofflichkeit}

Im Gespräch mit Frau Ryffel wurde oft das Wort «feinstofflich» bzw. «Feinstofflichkeit» verwendet. Wie kann man nun stofflich von feinstofflich abgrenzen, was kann man sich unter Feinstofflichkeit vorstellen?

Jacqueline Ryffel hat hier klare Vorstellungen:

Wenn beim Potenzieren ab D24 bzw. C12 der Verdünnungsgrad erreicht ist, der der Avogadro-Zahl entspricht, also $10^{23}$, dann sind sämtliche Moleküle der Ausgangssubstanz durch das Potenzieren eliminiert worden. Zurück bleibt nur noch die Energie und dies ist das Feinstoffliche. Wenn man eine $\mathrm{CD}$, die mit Musik bespielt ist, chemisch analysiert, dann kommen die identischen Resultate heraus wie bei der Analyse einer unbespielten CD. Die bespielte CD besitzt aber energetische Informationen zum Erklingen von Musik, die bei uns Emotionen auslösen kann. Oder: Wenn man in einem Büro sitzt und arbeitet, dann werden gleichzeitig eine Unmenge von SMS, WhatsApp-Nachrichten, E-Mails usw. in Form von elektromagnetischen Wellen durch diesen Raum geschickt. Wir sehen und hören aber nichts davon. Das ist sehr interessant! Wenn es möglich ist, z.B. einen kleinen Film innert Sekunden mithilfe von elektromagnetischen Wellen um die ganze Welt zu senden, dann ist es doch durchaus möglich, dass es ähnliche elektromagnetische Wellen gibt, die uns Menschen bei Beschwerden helfen.

\section{Unser Körper - eine Blackbox}

Solche Überlegungen zeigen, dass unser Körper in vielen Belangen immer noch eine Blackbox ist. Die ganze Medizin redet von Rezeptoren, die wichtige Funktionen erfüllen. Wer hat aber schon einmal einen solchen Rezeptor gesehen? Niemand! Und auch viele weitere Dinge in Bezug auf Gesundheit und Krankheit sind auf Theorien aufgebaut, welche die Vorgänge $\mathrm{zu}$ erklären versuchen. Viele dieser Theorien sind jedoch bis heute noch nicht bewiesen worden. Weiter kann man noch von keinem einzigen Medikament bzw. Wirkstoff den Weg und den Wirkungsmechanismus von der Einnahme bis zur Wirkung im Gewebe und in oder an den Zellen vollständig und in allen Details beschreiben.

Jacqueline Ryffel wörtlich: «Deshalb spielt es doch keine Rolle, wie eine Heilung zustande kommt. Auf naturwissenschaftlich erklärbare oder eben noch nicht erklärbare Weise. Letztlich hat doch derjenige recht, der eine Heilung erreicht hat!»

\section{Fachtitel \\ «Komplementärmedizin»}

Gibt es zwischen den komplementärmedizinischen Fachrichtungen Rivalitäten? Jacqueline Ryffel ist überzeugt, dass heute ein gegenseitiger Respekt besteht. Das ist vor 20 Jahren noch nicht der Fall gewesen, und heute spricht man sogar von einem Fachtitel «Komplementärmedizin». Darunter wird eine Ausbildung verstanden, die in verschiedene Therapierichtungen Einblick gibt. Das reicht dann aber noch nicht, um sich z.B. als Spezialist in Klassischer Homöopathie bezeichnen zu können. Dafür braucht es viel mehr Ausbildung als bei einem solchen allgemeinen Diplom. Und das gilt für die anderen Therapierichtungen auch. Dann kommt Frau Ryffel ins Schwärmen und meint: «Es wäre ja wunderbar, wenn jeder Hausarzt eine solche allgemeine Ausbildung in Komplementärmedizin hätte! Und wenn er dann merkt, dass jemand gut auf eine der Therapieformen anspricht, er aber selber nicht mehr weiterkommt, dann kann er ihn zum Spezialisten weiterschicken. Dies ist ja in der Schulmedizin mit ihren vielen Spezialdisziplinen auch der Fall.»

\section{Praxismodell der Zukunft}

Ein Praxismodell der Zukunft könnte so aussehen, dass verschiedene schulmedizinisch und komplementärmedizinisch tätige Ärzte in einer grossen Praxis zusammen tätig sind. Einer von ihnen, der von allem etwas versteht, macht bei einem neuen Patienten ein Screening und schickt ihn zu dem Arzt dieser Grosspraxis, bei dem ein Therapieerfolg am wahrscheinlichsten ist. Ein Diabetes wird dann z.B. schulmedizinisch behandelt und eine chronische Migräne komplementärmedizinisch. Die meisten homöopathisch tätigen Ärzte sind auch Allgemeinmediziner, die viele Beschwerden, z.B. einen Hypertonus, primär schulmedizinisch behandeln. Und wenn der Blutdruck gesenkt ist, dann kann dieser Arzt mit dem Patienten schauen, ob noch weitere Beschwerden vorhanden sind, die dann homöopathisch, phytotherapeutisch oder mit einer anderen Therapiemethode behandelt werden. 